\title{
La importancia estratégica de los Presupuestos Participativos en los gobiernos locales de Córdoba, Gualeguaychú, Paysandú y Montevideo
}

\section{The strategic importance of the Participatory Budgeting in the local governments of Córdoba, Gualeguaychú, Paysandú and Montevideo.}

Mariano Suárez**

\section{Resumen}

El presente artículo analiza la importancia estratégica de los presupuestos participativos en cuatro gobiernos locales de Argentina y Uruguay: Córdoba, Gualeguaychú, Paysandú y Montevideo. El análisis se focaliza en dos elementos, la posición del Presupuesto Participativo dentro de la estructura de gobierno, analizado en función del organigrama del mismo, y el alcance de la política en términos de la capacidad de la herramienta para generar transformaciones en el territorio. Este último aspecto se examinará sobre la base de entrevistas a responsables políticos, técnicos y ciudadanos. En el artículo se argumenta que las experiencias analizadas tienen una impronta más cercana a la función de movilización ciudadana que de gestión pública. Con relación a la posición de los Presupuestos Participativos, se observa que están más cerca de la estructura participativa que de las áreas de planificación y presupuesto, con la excepción de Montevideo, donde la condición de asesoría lo ubica en un lugar con mayor capacidad de transversalización. En lo que respecta al alcance, se trata de problemáticas muy micro que se perciben

\footnotetext{
* Artículo recibido 20 de septiembre de 2016. Aceptado 7 de febrero de 2017.

* Candidato a Doctor en Estudios Sociales de América Latina (CEA-UNC), Magíster en Desarrollo Humano (FLACSO- Argentina) y Licenciado en Ciencias Sociales (UdelaR). Integra el Grupo de Estudios de la Participación y la Descentralización, Universidad de la República, Sede Salto, Uruguay. Correo electrónico: marianodoc01@unorte.edu.uy
} 
como limitadas en su capacidad de nutrir otras áreas del gobierno.

\section{Abstract}

This article analyzes the strategic importance of Participatory Budgeting in four local governments of Argentina and Uruguay: Córdoba, Gualeguaychú, Paysandú and Montevideo. The analysis is focused on two elements, the position of the Participatory Budgeting inside the structure of the government, analyzed in terms of its organization, and the scope of politics as a tool to generate transformations in the territory. This last aspect is analyzed based on interviews with politicians, technicians and citizens. In the article it is argued that the analyzed experiences have a nearer relation to the function of citizen mobilization than the function of public management. In relation to the position of the Participatory Budgeting, it is observed that it is more related to the participative structure than the areas of planning and budgeting, with the exception of Montevideo where the condition of advising allows Participative Budgeting to be in a place with greater ability to influence transversely. With regard to the scope, there are micro problems that are perceived as limited to nourish other areas of the government.

\section{Palabras clave}

presupuesto participativo-coordinación-posición en el gobierno.

\section{Keywords}

Participatory Budgeting-coordination-position of the government.

\section{Introducción}

La última década del siglo XX y las primeras del siglo XXI han sido testigos de una revalorización de la idea de la participación ciudadana, la cual vino acompañada de importantes innovaciones en materia de programas participativos. En este escenario, la región latinoamericana ha tenido un papel protagónico, generando instrumentos participativos que luego fueron exportados al resto del mundo (Sintomer, 2008). Dentro de estos mecanismos, este artículo se centra en el Presupuesto Participativo (a partir de ahora PP) originado en Porto Alegre y hoy conocido como la experiencia emblemática de innovación democrática (Goldfrank, 2011), y practicado en todos los continentes del 
mundo (Molina, 2011). El objetivo central del trabajo es analizar la importancia que ha adquirido la política del PP dentro de la estrategia de los gobiernos locales de Córdoba, Gualeguaychú, Paysandú y Montevideo.

En los últimos años se advierte en la literatura especializada un interés creciente por el tema de la articulación entre este mecanismo de participación y las instituciones representativas (Schneider y Welp, 2011; Melo Romão, 2014; Montecinos, 2012; Rodgers, 2005; Gurza Lavalle, Melo Romão y Zaremberg, 2014, entre otros ejemplos). Esta articulación se puede dividir analíticamente en dos grandes aspectos, por un lado, la posición del PP en el gobierno y el alcance de las decisiones tomadas en él y, por otro, la mayor o menor influencia de las lógicas de los partidos políticos en la participación ciudadana. Este artículo se centrará en el primero de los ejes temáticos advirtiendo, sin embargo, que tanto la posición como el alcance del PP no son independientes del nivel de autonomía o subordinación de la participación con respecto a las lógicas políticopartidarias. Primeramente, se presentarán algunos de los principales aportes de las investigaciones vinculadas al tema. Posteriormente, se analizará, para las cuatro ciudades, la posición del PP en el organigrama del gobierno local, identificando cercanías - lejanías con las áreas de administración y planificación estratégica. Luego, sobre la base de los hallazgos de las entrevistas, se analiza el alcance de los PP en función de su capacidad para generar transformaciones en el territorio.

\section{Metodología}

El trabajo es parte de una investigación mayor que analiza los vínculos entre el PP y las tradicionales formas de representación político-partidaria, así como la forma en que este complementa o se subordina a las instituciones de la democracia representativa. La investigación original presenta un diseño cualitativo en el que se combinan diferentes técnicas y fuentes de información. En concreto, para este artículo, se han utilizado los aportes de las entrevistas a responsables políticos, técnicos y ciudadanos, y el organigrama oficial de los gobiernos locales. El proceso de investigación tuvo dos momentos de realización de entrevistas, una en el periodo electoral de 2015 y otra luego de que los cuatro gobiernos realizaran su primer año en el nuevo periodo de gestión. En este artículo se presentará únicamente el análisis de las entrevistas realizadas en el periodo electoral, en donde se buscó captar la percepción de los entrevistados con relación al funcionamiento de la herramienta en ese entonces y en los últimos años. En total se realizaron ocho entrevistas en Montevideo (un político, un técnico y seis ciudadanos) y siete en cada una de las otras ciudades (un político, un técnico y cinco ciudadanos).

Con relación al análisis del organigrama, se toma en cuenta tanto el del periodo anterior (2010-2015 en los casos uruguayos, y 2011-2015 en los casos argentinos) como 
el actual, poniendo de manifiesto los casos en los que ha habido transformaciones significativas en la posición de la política.

El análisis de los hallazgos se estructura en función de las siguientes dimensiones:

1. Alcance. Esta variable comprende tanto lo que tiene que ver con el diseño institucional como con el funcionamiento efectivo de la política. En ambos casos se evaluará la capacidad de la herramienta de generar transformaciones en el territorio, lo cual se operacionalizará en: a) la capacidad de impacto de los temas propuestos observada sobre la base de la magnitud del territorio en el que impactará, la población beneficiada, los montos de las propuestas aprobadas y la dispersión de propuestas o la generación de acuerdos que permitan reducir la cantidad de proyectos; b) la capacidad del PP para orientar otras políticas públicas, este punto se observa sobre todo en el tratamiento de los temas que no se aprueban dentro de esta política; y c) la coordinación entre el PP y las demás áreas del gobierno local; en este punto el interés se centra en el análisis de las instancias de comunicación y generación de acuerdos con otras áreas del Municipio de manera de no generar acciones descoordinadas con relación a un mismo punto y al hecho de facilitar o no los recursos de las diferentes áreas que son necesarios para implementar el PP.

2. Ubicación estratégica. Refiere al lugar ocupado dentro del organigrama municipal ¿De qué unidad o dirección depende su implementación? ¿Cuán cercana o lejana está esta unidad de los órganos de planeamiento y elaboración presupuestaria?

\section{Estado del arte}

\subsection{Alcance de las decisiones sometidas al PP}

La importancia con relación al impacto social y el alcance territorial de los temas abordados es sin duda uno de los aspectos que más ha despertado interés en lo que tiene que ver con el estatus del PP. La discusión de la literatura se centra sobre todo en la pertinencia de que en los PP se aborden discusiones y se elaboren proyectos sobre situaciones puntuales de la cotidianeidad de los vecinos 0 , por el contrario, se busquen articular propuestas de mayor escala capaces de pensar en problemas más abarcativos en términos de impacto y con una visión de desarrollo sobre la ciudad o la región.

En general, los autores no son demasiado afines a los PP circunscritos a problemas cotidianos, ya que dejan fuera de la discusión temáticas con mayor alcance social y político, y contribuyen, en ese sentido, a la subordinación del PP a las instituciones de la democracia representativa (Montecinos, 2009 y 2012; Cabannes 2004 y 2005, entre otros). Basado en un estudio empírico de 13 casos chilenos, Montecinos (2009) sostiene que en los problemas barriales los ciudadanos prefieren tomar parte activa de la decisión, en cambio en los problemas de la ciudad tienden a delegar las decisiones a los 
representantes. Otro elemento, que para el autor contribuye a la subordinación, es el hecho de que el PP es entendido como un programa y no se enmarca en una política de participación ciudadana en donde se generen espacios de coordinación con otros mecanismos de participación. Sumado a esto, Montecinos (2009) advierte en los casos estudiados el excesivo protagonismo del gobierno tanto en la definición del diseño institucional como en la definición de ciertas temáticas que en muchos casos parecen expresar más un interés del municipio que de la ciudadanía.

Por su parte, Rocío Annunziata (2011 y 2013) constata un cambio en la legitimidad democrática que implica un deslizamiento hacia la proximidad, ambientando de esta forma el surgimiento de programas participativos y un conjunto de prácticas y discursos con una tendencia hacia la singularidad del espacio y de los problemas. El primero se vuelve local y cotidiano; los segundos se ligan fuertemente a las vivencias personales. Relativo a los PP se muestra cómo en los casos de Rosario y Morón hay una clara preferencia ciudadana hacia muchos proyectos pequeños; se sostiene, a su vez, que la fragmentación de las propuestas debilitan la herramienta en términos de su carácter político y redistributivo. En este sentido, se argumenta que, en estos dos gobiernos locales progresistas con voluntad de promover la participación y pioneros en la implementación del PP en Argentina, se genera, sin embargo, una participación más ligada a la gestión del entorno inmediato y marcada por la fragmentación. La autora concluye que no se puede asumir que a nivel local la participación no tiene carácter político y recomienda incluir este componente, que no depende solamente de la orientación progresista del gobierno, sino también del diseño institucional de la política y de las prácticas de los actores involucrados en el proceso (Annunziata, 2013).

\subsection{Posición del PP y coordinación con otras estructuras del gobierno}

La ubicación del PP dentro del organigrama de gobierno marca una mayor cercanía o distancia con las diferentes entidades que conforman el gobierno. Para Melo Romão (2014), esta ubicación tiene consecuencias sobre los vínculos que se establecen entre el PP y los otros órganos de la administración, marcando de esta manera un determinado perfil de la política en cuanto a la función que esta cumple dentro del gobierno. El autor analiza cuatro experiencias de PP en el Brasil (Diadema, Osasco, San Bernardo del Campo y Guarulhos), en donde concluye que:

1) El estatus político del PP está vinculado con la localización en la administración municipal. En este aspecto se mencionan tres posibilidades: a) cerca de la estructura de planeamiento y elaboración presupuestaria; b) en medio de la estructura de planificación de la prefectura; y c) lejos de la planificación y sin vínculos visibles con las estructuras de planificación. 2) Cuanto mayor es la presencia de miembros del gobierno en los Consejos de PP, hay más posibilidades de generar decisiones políticas reales sobre presupuesto. 3 ) 
El perfil de los consejeros de la sociedad civil en el PP es más cercano a la coalición de gobierno cuanta más efectividad decisoria hay en el Consejo del PP.

El autor, a su vez, propone una tipología de funciones del PP. En ella se sugiere que el PP tendrá un rol clave en el planeamiento cuando se encuentre más cercano a oficinas de planificación y la distribución de participantes en el Consejo -entre sociedad civil y gobierno- sea equitativa, pero los consejeros sean cercanos al gobierno. En cambio, el PP tendrá un rol central en la estrategia de movilización de militantes y fuerzas políticas con afinidad hacia el gobierno cuando la coordinación del PP esté más vinculada a la unidad de participación y no a la de planificación, y hay un mayor número de participantes de la sociedad civil en el Consejo. En estos casos, el PP tiene una función más cercana a establecer un diálogo con la ciudadanía que a la toma de decisiones.

Por su parte, Yanina Welp (2015) entiende que este trabajo de Melo Romão conduce a sugerir que el PP funciona mejor cuando se da una relación de mutuo beneficio entre los promotores -el gobierno- y los ciudadanos más cercanos a las políticas del mismo, en cambio cuando se da la situación contraria se desnaturaliza y pierde sentido. O sea que el PP es más poderoso cuando más subordinado es al proyecto del partido en el gobierno.

Otro aspecto importante para dotar al PP de una visión de desarrollo es el establecimiento de canales de comunicación entre este y otras políticas públicas, sean estas mecanismos representativos o participativos. Esto aumentaría el potencial del PP de influir en la orientación de estas políticas (Montecinos, 2012; Welp, 2015). En este sentido, Welp (2015) señala que cuando las deliberaciones se vinculan a la toma de decisiones dentro del marco de planes estratégicos de desarrollo, los temas que orientan el debate tienden a ser más sustantivos; la autora menciona en este sentido los casos de Bogotá, Quito y Montevideo. El caso contrario vendría dado cuando la participación carece de estos marcos normativos para orientarse y la tendencia sería hacia temas puntuales de poca relevancia. En este último caso, el ejemplo podría ser Buenos Aires.

\section{Análisis de los casos}

\subsection{Presentación de los casos y posición del PP en el organigrama de gobierno}

\subsubsection{Paysandú}

La ciudad de Paysandú es capital del departamento homónimo con una población de 74.575 habitantes. El PP se originó con la llegada del Frente Amplio (de orientación progresista) por primera vez al gobierno de la ciudad en el año 2005, de la mano de Julio Pintos. La Intendencia, en ese periodo, creó la Dirección de Descentralización y dentro de ella, una unidad de la política que se transformaría en el buque insignia de su oferta 
participativa, el PP. Luego de cinco años de ejecución de la experiencia, el Frente Amplio perdió el gobierno frente al Partido Nacional (asociado a la centro derecha), encabezado por Bertil Bentos, quien en un principio no se mostró afín a esta política. Sin embargo, decidió continuarla, pero con una serie de modificaciones en la metodología. En la actualidad ha vuelto el Frente Amplio al gobierno y se está procesando una nueva serie de modificaciones metodológicas.

Con relación a la metodología utilizada en el PP de Paysandú, se puede decir que se trata de una convocatoria anual a presentación de proyectos por parte de ciudadanos y organizaciones. En cualquier caso se debe contar con el apoyo en firmas de al menos cien vecinos que respalden el proyecto. Se realizan dos asambleas obligatorias para quienes quieren presentar iniciativas. Luego, las propuestas son sometidas a un análisis de facitibilidad realizado por técnicos de la Intendencia. Y posteriormente se realiza una votación con todos los proyectos viables que está organizada por distrito y por temáticas. Es decir, los proyectos compiten dentro de uno de los ocho distritos y dentro de una de las seis temáticas: obra/servicio departamental, inclusión social, discapacidad, educación, salud y juventud. A través de la votación se genera un ordenamiento y se financia siguiendo el mismo hasta agotar los montos del distrito y el tema. La ejecución se hace a través de mecanismos de cogestión con traspaso a las organizaciones de la sociedad civil.

Como puede observarse en la figura I, en la ubicación del PP en el organigrama del gobierno de Paysandú se destacan dos elementos importantes. Uno es que el PP asume la forma de una unidad, dentro de una dirección que depende de un departamento vinculado a la Secretaría General y al Intendente. Es decir, la jerarquía del órgano que lo implementa es la más baja en el organigrama departamental, lo cual para un programa que en su ejecución implica el desarrollo de actividades complejas, pertenecientes a rubros muy diversos del quehacer de la Intendencia, puede generar algún tipo de dificultad a la hora de conseguir la colaboración de las otras áreas -en donde se debe solicitar el apoyo de jerarcas de más rango-, sin la cual sería imposible la ejecución de esta política polirubro. 


\section{Figura I. El PP en el organigrama del gobierno de Paysandú}

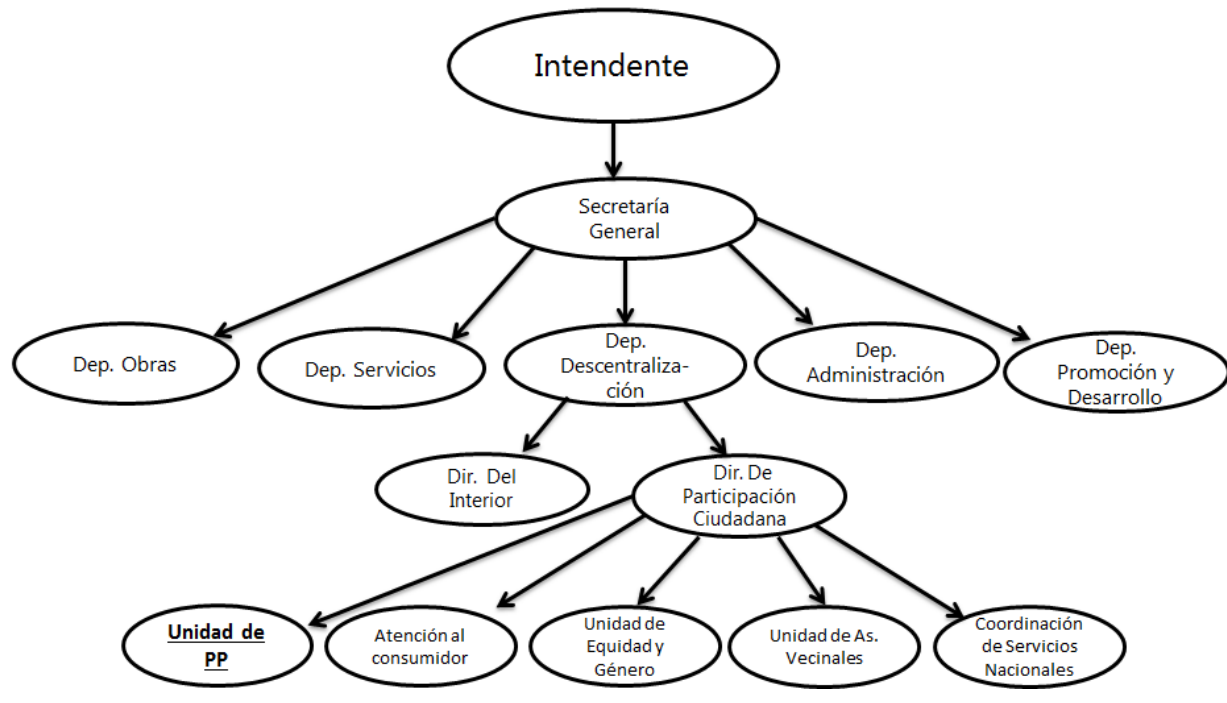

Fuente: Elaboración propia

El segundo elemento a destacar es que, siguiendo el análisis de Melo Romão (2014), se puede decir que el PP en Paysandú tiene una impronta clara de movilización más que de planeamiento, ya que se encuentra dentro del Departamento de Descentralización y más específicamente dentro de la Dirección de Participación Ciudadana, generando en el esquema muy poca conexión con la estructura de toma de decisiones sobre presupuesto y planeamiento. Por el contrario, las cercanías mayores del programa tienen que ver con Unidades de asuntos vecinales y ciudadanos. Dentro del Departamento de Descentralización se desprende, por un lado, la Dirección del Interior, que articula la actividad del segundo con el tercer nivel de gobierno y, por otro lado, la Dirección de Participación Ciudadana en donde además del PP, se atienden programas vinculados a la equidad de género, atención al consumidor, comisiones vecinales y servicios nacionales. En resumen, la participación en su conjunto, y el PP dentro de ella, no se ubica en un lugar que tenga conexión directa con la estructura de toma de decisiones presupuestales u otras áreas de generación de políticas públicas.

\subsubsection{Montevideo}

Montevideo es la capital del país con una población de 1.319.108 habitantes. Con la llegada del Frente Amplio por primera vez a la Intendencia en 1990 junto a Tabaré Vázquez, el gobierno de la ciudad inició un proceso de descentralización que sería el marco general dentro del cual se produjo el funcionamiento del PP. Esta política fue puesta en marcha por primera vez en el año 1990, siendo de esta forma una de las experiencias pioneras en América Latina y el mundo. Con el tiempo llegó a transformarse en una experiencia emblemática en la región, estando actualmente en el sexto quinquenio de ejecución, todos ellos en manos del Frente Amplio. El PP de Montevideo ha ido pasando por diferentes metodologías, las cuales otorgaban más o menos 
protagonismo a los Consejos Vecinales (órganos de representación vecinal creados en el proceso de descentralización), manteniendo, sin embargo, su carácter de bajo nivel de institucionalización y siendo regulado, únicamente, por un reglamento interno.

Actualmente, el diseño implica una convocatoria bianual a presentación de proyectos que puede hacerse de manera individual o por organizaciones. La base territorial son los ocho municipios de la ciudad de Montevideo. Las temáticas deben enmarcarse en alguna de estas grandes áreas: social, cultural u obras. Para la evaluación de factibilidad en cada zona, se conforman equipos técnicos que trabajan, a su vez, en coordinación con equipos de planificación de cada municipio y con la Unidad de Planificación y Participación de la Intendencia Departamental de Montevideo (IDM). La metodología actual no implica reuniones asamblearias de ciudadanos y la forma de dirimir qué proyectos se financiarán es a través del voto universal y secreto. Hay un año destinado a la elaboración y elección, y otro para la ejecución. Dicha ejecución está a cargo del Ejecutivo departamental y los municipios. Antes de iniciar un nuevo proceso participativo, se realiza una instancia de evaluación y modificación de reglas vigentes en las que participan los Consejos Vecinales.

De los casos analizados, el de Montevideo es el que muestra una posición más estratégica para el aprovechamiento de la participación en el PP como insumo para otras áreas del gobierno departamental.

Figura II. EI PP en el organigrama del gobierno de Montevideo

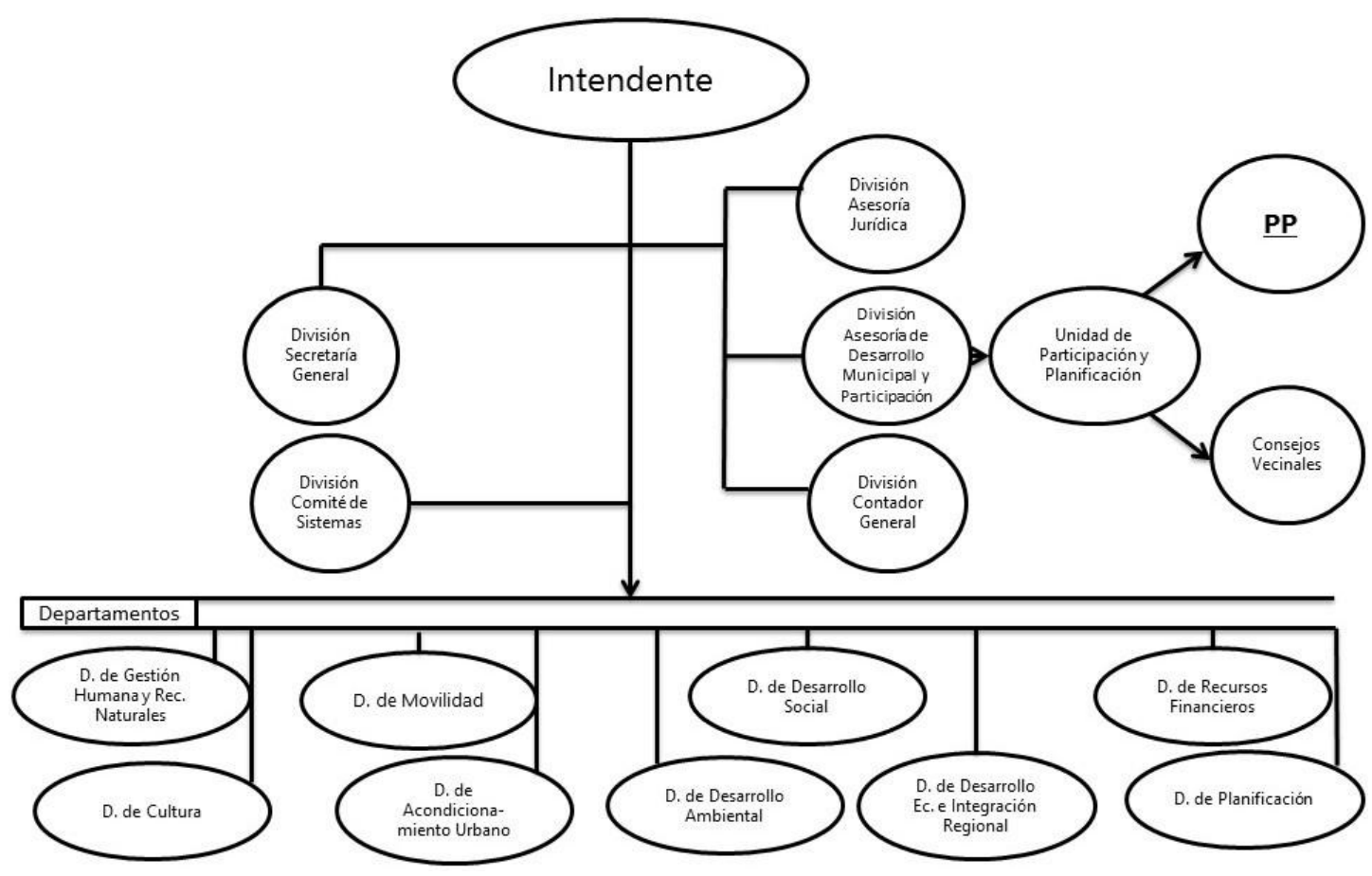

Fuente: Elaboración propia 
Al observar la figura II, puede verse que el PP depende de una asesoría que dialoga directamente con el Intendente. Dicha asesoría, de la que depende, es la de Desarrollo Municipal y Participación, lo cual muestra una conjunción entre la planificación del desarrollo y la participación. A su vez, al estar por fuera de las divisiones departamentales, tendrá, al menos en el diagrama, una perspectiva transversal, es decir, tanto las definiciones de desarrollo municipal como la participación ciudadana estarían transversalizando la gestión de las diferentes áreas departamentales. Esto es un aspecto fundamental y distintivo de Montevideo, que no coloca la participación como un área departamental específica desenganchada de los demás temas, sino que al formularse como asesoría tiene, en términos de posición, un lugar mucho más estratégico para nutrir las demás agendas del quehacer del gobierno departamental. Dentro de la Asesoría de Desarrollo Municipal y Participación, el PP está ubicado en la Unidad de Participación y Planificación que tiene entre sus objetivos principales apoyar a los gobiernos municipales en la elaboración de sus planes de desarrollo, fortalecer el PP y promover la acción colectiva organizada. El PP, a su vez, dialoga con los Consejos Vecinales que también se mueven dentro de esa órbita.

\subsubsection{Gualeguaychú}

La ciudad de Gualeguaychú es la capital de un departamento con el mismo nombre, ubicado en la provincia de Entre Ríos (Argentina). El municipio comprende la ciudad y un área rural, sumando en total una población aproximada de 83.116 habitantes. Su actual intendente es Martín Piaggio, quien sucedió a Juan José Bahillo, ambos del Frente para la Victoria (fuerza política de centro izquierda). En el periodo anterior, en el año 2012, comienzó a funcionar el PP con una dinámica muy cercana a las metodologías brasileñas en donde se desarrollaron varias rondas deliberativas en una convocatoria abierta que buscaba llegar a acuerdos sobre las propuestas a financiar.

A grandes rasgos, la metodología del PP consiste en una convocatoria anual a presentación de propuestas que pueden hacerse de manera individual o través de organizaciones; en cualquier caso, debe haber un respaldo de al menos veinte ciudadanos y en caso de no haber organización que respalde, la ejecución se hará a través de la Comisión Vecinal. El proceso implica cuatro reuniones asamblearias, organizadas en nueve distritos, en donde se presentan y se van discutiendo las propuestas. En la reunión final, se busca llegar a un consenso entre los ciudadanos presentes sobre qué proyectos financiar teniendo en cuenta los montos máximos por distrito. En esta reunión se puede modificar el alcance de las propuestas para ajustarlo a los montos disponibles. En caso de no llegar a un acuerdo entre los presentes, se prevé una votación universal y secreta para ese distrito. La ejecución está a cargo de la Intendencia, quien en caso de tratarse de obras, contrata las empresas para la realización. 
El análisis del organigrama del caso de Gualeguaychú se debe separar en dos periodos, ya que el nuevo gobierno, iniciado en diciembre de 2015, realizó modificaciones que afectaron directamente la posición del PP en el esquema.

Figura III. EI PP en el organigrama del gobierno de Gualeguaychú (2011-2015)

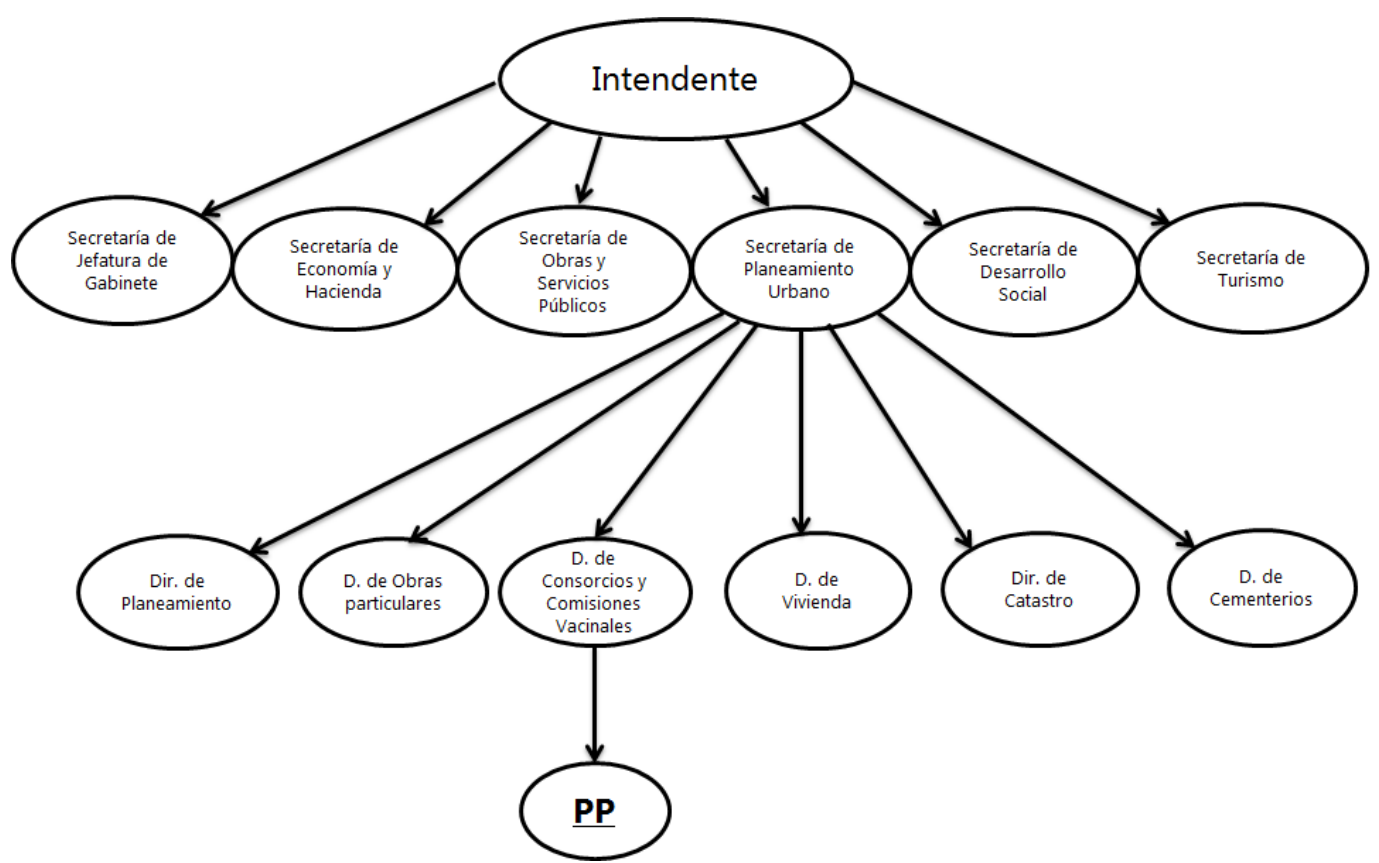

Fuente: Elaboración propia

Figura IV. EI PP en el organigrama del gobierno de Gualeguaychú (2015-2019)

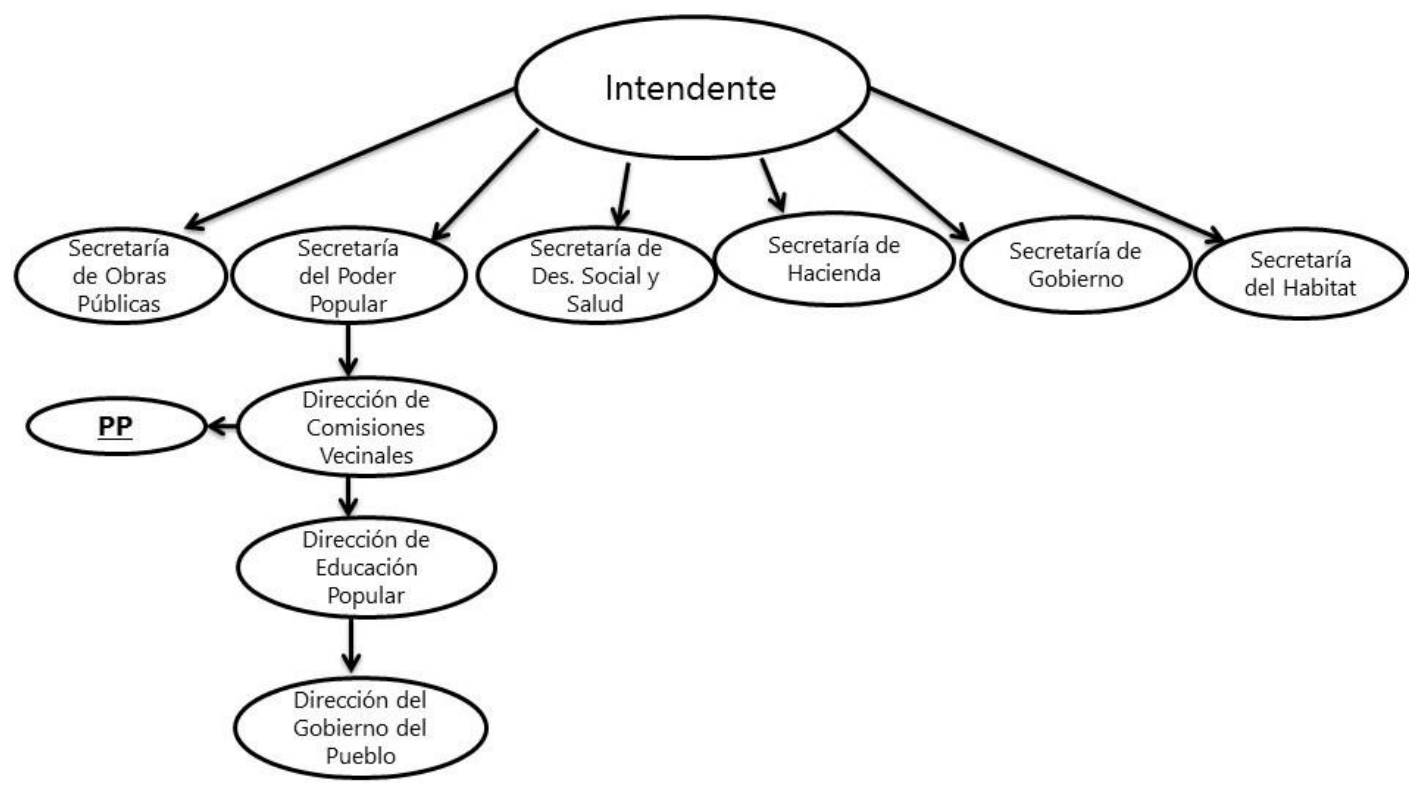

Fuente: Elaboración propia 
Como se puede observar en la figura III y IV, tanto en el periodo de gobierno anterior como en el actual, el PP aparece en una posición baja en la cadena jerárquica. En la actualidad, se ubica como un programa que está dentro de una dirección (de Comisiones Vecinales), la cual depende de una secretaría (de Poder Popular).

Por otro lado, con relación a las conexiones que establece el PP, se puede observar que la dirección de Poder Popular fue creada para este nuevo ejercicio de gobierno y tal como lo muestra la figura IV, nuclea todas las direcciones -y sus respectivos programasreferidas a la participación ciudadana. La impronta del PP es, en este sentido, claramente orientada hacia la movilización de actores sociales, estando la parte de planificación y administración ligada a otras secretarías. Como puede observarse en la figura III, en el periodo anterior, el PP formaba parte de la Dirección de Consorcios y Comisiones Vecinales, la cual dependía de la Secretaría de Planeamiento Urbano. A partir de esta nueva estructura, toda la plataforma de programas participativos pasó a tener un estatus de secretaría, y el PP se corrió junto con ellos. De manera que el PP siempre estuvo más cerca de la estructura de participación que de la de presupuesto, aunque dependiendo de la Secretaría de Planeamiento Urbano, la participación en su conjunto adquiere una posición más estratégica para dialogar con aspectos de planificación, en este caso, de planificación urbana, que es un área vinculada a buena parte de los proyectos presentados en el programa.

En este periodo de gobierno, iniciado a fines del año 2015, los programas participativos adquirieron un estatus mayor al conseguir posicionarse como secretaría, pero el PP se mantuvo como un programa dentro de una dirección y al acompañar a los programas participativos hacia otra secretaría, se alejó en el organigrama de los aspectos de planificación estratégica. No se avizora, en el esquema, una capacidad de influencia de los programas de participación hacia otras áreas de la actividad de gobierno.

\subsubsection{Córdoba}

La ciudad de Córdoba es la capital de la provincia homónima y cuenta con aproximadamente 1.329 .604 habitantes, y con una gran dispersión territorial. En el año 1991, el gobierno de la ciudad comenzó un proceso de descentralización y desconcentración administrativa. En este marco, las políticas de participación ciudadana estuvieron ancladas sobre los Centros de Participación Comunal (CPC), a través de las Juntas de Participación Vecinal, siendo el PP uno de los principales instrumentos de deliberación y toma de decisión ciudadana. La relación entre las Juntas de Participación Vecinal y el PP es un aspecto clave que por momentos generó tensiones y determinó el carácter plural $u$ homogéneo en la participación de este último. Tanto las Juntas de Participación Comunal como el PP fueron regulados a partir de 2008 por sus respectivas ordenanzas. Sin embargo, el PP surgió de manera menos formal en el periodo de 
gobierno comprendido entre 2003 y 2007, encabezado por Luis Juez, del Partido Nuevo (de orientación progresista), en alianza con otros partidos.

Su metodología implica una convocatoria anual a presentación y selección de propuestas. Hay dos espacios territoriales para la articulación del diagnóstico participativo: la Zona Barrial y los Centros de Participación Comunal (CPC). Dentro de los primeros se realizan convocatorias abiertas a todos los vecinos con el fin de realizar un diagnóstico de la zona, definir prioridades y elegir delegados para el Taller del PP. En los CPC, que tienen una escala mayor reuniendo varias Zonas Barriales, se realiza el Taller de PP en donde solo asisten delegados electos previamente y junto con las autoridades municipales definen cuáles serán las propuestas - presentadas en las etapas anteriores- a financiar en ese CPC. La ejecución está a cargo del Municipio, generándose una etapa de control de la ejecución por parte de los ciudadanos a cargo de las Juntas de Participación Vecinal y los delegados electos para el taller de PP.

Con relación al análisis de la posición del PP, de los cuatro casos analizados, el de Córdoba es el que posiciona la política en una mayor jerarquía, ya que el PP es en Córdoba una dirección, la cual tiene por encima la Subdirección de Participación Ciudadana, perteneciente a la Secretaría de Gobierno Participativo y Desarrollo Social.

Figura V. El PP en el organigrama del gobierno de Córdoba (2015-2019)

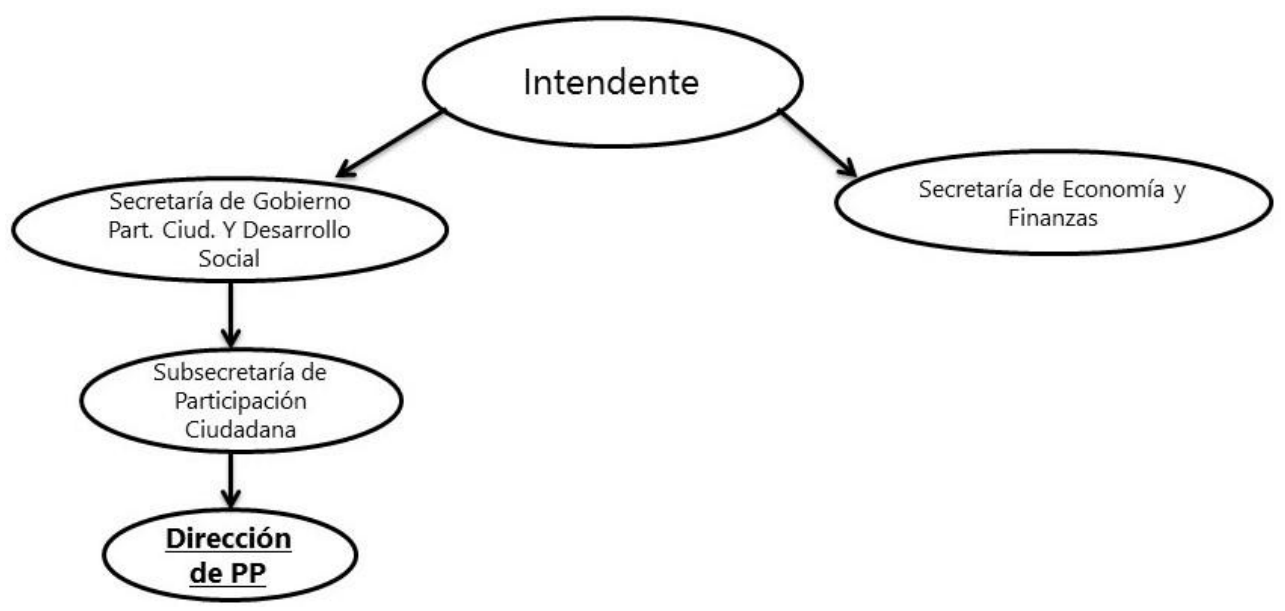

Fuente: Elaboración propia

Como se ve en el esquema $\mathrm{V}$, la temática de economía y finanzas pertenece a otra secretaría, por lo cual, se puede decir que el PP no está pensado como un espacio de influencia en términos de definición de una estrategia de gasto público. Sin embargo, se puede observar a la participación ciudadana en la misma secretaría que el desarrollo social, esto implica que el PP tendría posibilidades de dialogar con las políticas de desarrollo social aplicadas en el municipio. Como se ha dicho en el caso de 
Gualeguaychú, en el periodo 2011-2015, el PP tenía contacto directo con el área de Planeamiento Urbano propiciando así el diálogo con esta área, que maneja una temática muy recurrente dentro de los proyectos aprobados en la política. En Córdoba, no obstante, se lo acerca al área de Desarrollo Social, que también es un área fuerte en la mayoría de los PP. Este es un aspecto interesante que permite analizar el sentido con el que se piensa la política en un caso y otro.

\subsection{Alcance del PP en las diferentes experiencias}

\subsubsection{Paysandú}

Con relación al tipo de temáticas que se propone en los PP, como se ha dicho en la revisión literaria, una de las discusiones más recurrentes tiene que ver con la pertinencia de que en los PP se elaboren propuestas sobre situaciones puntuales de la cotidianeidad de los vecinos o, por el contrario, se busquen articular propuestas de mayor escala, capaces de pensar en problemas más abarcativos en términos de impacto y con una visión de desarrollo sobre la ciudad o la región. En este sentido, es posible identificar dos enfoques en los diseños de los PP. Por un lado, el enfoque territorial, en donde se trata de resolver aspectos puntuales de cada uno de los territorios en los que se divide la aplicación del programa y los fondos generalmente se dividen en partes iguales entre los diferentes territorios. El otro enfoque es temático, en él se trata de pensar la ciudad en su conjunto, se contemplan cuestiones transversales que exceden el territorio de pertenencia inmediata de los vecinos (Martínez y Arena, 2011).

En el caso de Paysandú, al igual que en los demás casos analizados en este artículo, se trata de un PP con enfoque territorial, en donde si bien se plantean algunos ejes temáticos, estos son utilizados para excluir algunas problemáticas y para combatir el efecto de las inequidades en la competencia, pero siempre desde una lógica territorial micro- y no de ciudad. Uno de los puntos más destacados en el análisis de las temáticas es que la experiencia de Paysandú sigue la tendencia que en general se ve en los PP, la cual marca que estos se vinculan a pequeñas transformaciones en escalas territoriales pequeñas (Montecinos, 2009). En general, se advierte una visión positiva con relación a este punto y si bien aparecen en los discursos algunas voces, marcan la necesidad de aumentar los montos e incluso en algún caso se habla de modificar el diseño de manera de que ganen menos proyectos, pero de más porte. Sin embargo, aún en estos casos no se está pensando en proyectos de interés general para la zona o la ciudad, sino en intereses particulares que afectan a un determinado grupo de personas, de una escala pequeña, como un club, una escuela, una asociación para la atención de la discapacidad $u$ otras. Es decir, el cuestionamiento no es hacia las temáticas micro, sino hacia el hecho de que esas problemáticas necesitan de sumas más elevadas para resolverse y eso no se está logrando con la metodología o con los montos actuales. 
bueno, siempre se puede mejorar, dar un poquito más, agregar un poquito más, porque ahora nomás, a nosotros no nos va a dar ni para empezar, pero siempre es una ayuda (participante del PP de Paysandú).

Yo, en lo particular, creo que habría que repartir entre menos proyectos y más cantidad de dinero. Es decir que si vos ganás un año, el PP te dé para financiar tu proyecto de forma contundente. Aunque no te puedas presentar al otro año (participante del PP de Paysandú).

Como se ha visto, este no es un tema novedoso, sino una tendencia bastante general. Así lo advierte Montecinos (2011), quien explica para los casos chilenos que cuando se discuten temas de la ciudad, la participación cae en intensidad y asumen un rol más protagónico los representantes, direccionando algunas temáticas de los PP más hacia temas reducidos que a temas de interés general. Se puede decir que los diseños llevan intencionalmente a ese tipo de cuestiones, pero, a su vez, que la gente, en general, tiende a perder interés a medida que se aumenta la escala de trabajo y los impactos se vuelven menos tangibles.

Otro aspecto importante es que el diseño excluye determinadas temáticas y organiza la competencia territorial en función de cinco ejes: 1) obra/servicio departamental, 2) inclusión social, 3) discapacidad, 4) educación, 5) salud y 6) joven. De esta forma, se asignan determinados montos a cada tema, por cada distrito de la ciudad, y se realizan competencias electorales entre propuestas. Si bien en las instancias de asambleas puede haber acuerdos entre organizaciones que deciden no competir y dividir los montos, los acuerdos planteados no van en la dirección de atacar una problemática común que se lleve todos los fondos, sino en dividir el dinero y llevarse un poco cada uno.

Lo de las alianzas es bueno, es bueno porque nos ayudamos un poquito para cada uno, pero me gustaría que fuera un poquito más de plata (participante del PP de Paysandú).

Nosotros ganamos en ese año el primer y el segundo premio, cuatrocientos cincuenta mil pesos por cada uno, ahora es todo distinto, es por temática, y este año en salud creo que fue lo mínimo, ciento cuarenta mil pesos para dividir entre dos (participante del PP de Paysandú).

Pese a los montos bajos, que fueron resaltados de forma unánime en los discursos, llama la atención un aspecto, que también aparecerá en algunos casos de Montevideo, y es que muchas organizaciones sociales se vuelven dependientes del PP para desarrollar su actividad. Es decir, que para ellos ganar el PP significa obtener el presupuesto con el que deberán trabajar durante el año.

es re importante, porque nosotros toda la rehabilitación que hacemos es gracias a lo que hemos podido comprar con ese dinero, el pago de la fisioterapeuta, parte del pago de la 
camioneta que nos traslada. Lo que tenemos es gracias al PP (participante del PP de Paysandú).

Te genera esa dependencia, nosotros somos PP dependientes, esto funciona porque está el PP, sino no funciona (participante del PP de Paysandú).

O sea que hay determinadas organizaciones que participan desde el principio en el PP, para las cuales no sería posible sobrevivir sin esa ayuda, lo cual, por un lado, hace que se valore la herramienta, pero, por el otro, es claro que se trata de un funcionamiento precario que necesita, en algunos casos, de programas que les garanticen una financiación más estable que les permita planificar en un plazo mayor la actividad.

Al analizar la percepción sobre la coordinación en el PP de Paysandú, se advierte en el responsable político una valoración hacia la necesidad de coordinar entre áreas, pero señala con claridad la dificultad de generar estas articulaciones. Por un lado, el PP para muchos sigue identificado a la gestión del Frente Amplio, que fue quien lo creó, y, en ese sentido, se puede advertir un desinterés por potenciar una herramienta que entienden no les traerá réditos políticos; pero, por el otro, las propias áreas están cargadas de trabajo y en ese sentido los apoyos al PP significan una carga extra.

porque la administración, una cosa que le costó muchísimo es hacer la coordinación entre las diferentes direcciones; hablarlo acá sentados es facilísimo, concretarlo en la realidad es imposible, por todo el tiempo que lleva y porque están todos llenos de sus propias actividades, entonces no hay coordinaciones (responsable político del PP de Paysandú).

Estos problemas de coordinación llevan a que desde la dirección de PP se piense en la necesidad de contar con un área técnica propia que les permita desarrollar de forma autónoma y con calidad los estudios de viabilidad técnica. De todas formas, esto no solucionaría el problema de la coordinación, pudiendo generarse superposición de inversiones, entre otros problemas.

Finalmente, en el punto vinculado a la capacidad de orientar políticas públicas, se puede decir que en Paysandú el PP no cumple esa función, su contribución se remite a la resolución de los problemas puntuales que fueron aprobados, sin generar soluciones a los que no fueron aprobados y nutrir adecuadamente las áreas de la Intendencia con las demandas y propuestas ciudadanas.

Sería bueno que el PP dialogara con otros ámbitos, incluso fuera de la Intendencia, para buscar soluciones más profundas y que generen menos esa dependencia de ganar todos los años. Eso es lo que está faltando, una articulación (participante del PP de Paysandú).

en relación a los proyectos que no salían, nosotros tenemos una Unidad Vecinal, pero al no tener recursos, quedamos siempre sujetos a que hay que hacer una articulación. Esta 
área debe transformarse en lo que puede ser una Dirección de Participación Ciudadana que a esos proyectos los tome (...) (responsable político del PP).

En los discursos que anteceden queda claro la existencia de esta falencia y la importancia de avanzar en términos de un mayor aprovechamiento de la participación. Aun cuando se trate de demandas puntuales, estas pueden dar un mapa de las problemáticas de las zonas a partir de las cuales pensar en planes y programas más generales.

\subsubsection{Montevideo}

En lo que respecta a las temáticas que se presentan y que se aprueban en el PP de Montevideo, resaltan dos aspectos centrales en materia de diseño. Por un lado, no existen limitaciones con relación a las temáticas sobre las que se pueden presentar proyectos. Y por el otro, el proceso no prevé en ningún momento instancias de deliberación en las cuales los ciudadanos puedan acercar puntos de vista o generar acuerdos sobre problemáticas comunes. El resultado es una gran dispersión de temas que no alcanzan a tener una gran envergadura. Se trata, por tanto, de un modelo de corte más consultivo (Montecinos, 2011), en donde el papel central del grueso de los ciudadanos es indicar las prioridades a través del voto universal y secreto.

La tendencia a generar muchos proyectos con un alcance micro está definida en el tipo de diseño que se propone que, por un lado, tiene un enfoque territorial y no temático, y por el otro, se centra en la competencia democrática y no en la generación de acuerdos. Desde el gobierno se señala que el objetivo principal del PP es crear conciencia ciudadana y, en ese sentido, la idea de crear una conciencia departamental que les permita pensar la ciudad en su conjunto, con sus problemáticas centrales, no se ha constituido en una prioridad para ellos.

Muchas veces se ha pensado que hubiera dos, o tres, o cuatro propuestas de alcance departamental, pero no, nunca lo hemos visto como algo central. Porque el tema central es la creación de conciencia ciudadana, la participación, se puede decir, pero también estaría bueno generar una conciencia departamental, sí estaría bueno como tanta cosa, como presupuesto infantil, de jóvenes, etc. (cargo político en el PP de Montevideo).

De manera que las propuestas son referidas a problemas que involucran a actores concretos más que a zonas o a la ciudad; en ese sentido, pueden ser evaluadas externamente como de poco impacto, sin embargo, los ciudadanos participantes no lo visualizan como propuestas de poco impacto, ya que en muchos casos a pesar de no ser grandes inversiones, tienen impactos sociales que ellos valoran como importantes. En este aspecto se distinguen dos elementos con relación a Paysandú que en algún sentido explican esta visión, los montos son mayores y las instituciones u organizaciones deben cumplir determinadas contrapartidas que amplían el espectro de los beneficiarios. El otro 
elemento interesante es que, como se ha dicho en el análisis de la experiencia de Paysandú, para algunas organizaciones el PP es clave para lograr el desarrollo de su actividad.

En los discursos analizados puede observarse una tensión en torno a si es pertinente mantener la amplitud que habilita a presentar cualquier tipo de propuestas o si habría que generar algunas restricciones para mejorar el potencial de la herramienta. Las principales discusiones pasan por la inclusión o no de obras de infraestructura y de tareas que debería realizar la Intendencia como parte de sus obligaciones como gobierno. El argumento central para dejar fuera estos temas es que el PP tendría que abordar temas que emerjan de la creatividad social y no señalar temáticas que la Intendencia ya sabe que debe cumplir. Hasta ahora se ha impuesto la idea de permanecer con el criterio de máxima amplitud por entender que a pesar de que a veces puede dar lugar a propuestas poco creativas, estas pueden apuntar a necesidades elementales de las poblaciones más carenciadas.

La idea de infraestructura sí o infraestructura no, como semáforos, etc., se ha laudado por el hecho de que las zonas que tienen más carencias plantean que es como un egoísmo de quienes lo plantean, de no permitirlo, porque en realidad para ellos es importante, porque tienen muchas necesidades para cubrir en ese rubro (...) (participante del PP de Montevideo).

La idea que subyace es que en la medida que se satisfacen las necesidades más elementales, como saneamiento, cordón cuneta y otros, tienden a emerger otro tipo de demandas que son las que reivindican los sectores que no se encuentran en esta situación de privación. Este aspecto es advertido por Welp (2015), quien, haciendo una mirada general de los PP, sostiene que las decisiones que son posibles de tomar a través de esta herramienta tienen un marcado carácter compensatorio y que este aspecto tiene sentido en contextos de elevada deprivación, pero comienza a perder sentido en escenarios donde las necesidades más básicas están resueltas. Esto permite entender el posicionamiento, tanto de quienes quieren ir más lejos quitando determinadas temáticas como de quienes consideran egoísta este planteo.

Por otro lado, se argumenta que el hecho de que sean tareas de la Intendencia no significa que esta fuera hacerlas, ya que la lista de prioridades de cada área del gobierno departamental no tiene por qué coincidir con la de la ciudadanía y ese es justamente el potencial de la herramienta, poder colocar como prioridad algo que para el gobierno no lo era, como se expresa en este discurso: "en nuestro barrio ganó un semáforo la elección, la ganó por lejos, en una esquina que para la administración municipal debía estar en un lugar doscientos en la lista de prioridades, pero para nosotros era la número uno" (participante del PP de Montevideo). 
El otro aspecto que genera discusión tanto dentro de la ciudadanía como en la interna del gobierno tiene que ver con el hecho de financiar proyectos que involucren a espacios privados como, por ejemplo, los clubes, cuyo acceso es restringido a una determinada población, los socios. En este punto, el gobierno define permitir ese tipo de inversiones, pero determinando contrapartidas que implican generar espacios en los cuales ese beneficio pueda ser utilizado por la comunidad en general. Como se ha dicho, este aspecto contribuye a mejorar el impacto social de las propuestas.

En lo que respecta a los aspectos de coordinación, al ser Montevideo una propuesta que ya está muy instalada, tiene bastante desarrollado algunos mecanismos de contactos en las diferentes áreas. Además, como se mencionó más arriba, la ubicación en el organigrama como una asesoría que transversaliza los diferentes ejes le es favorable a los efectos de compartir información. De acuerdo con lo expresado por los funcionarios del PP entrevistados, la coordinación asume sobre todo tres mecanismos estables.

Por un lado, todas las áreas de la Intendencia asignan a uno de sus funcionarios para acompañar el proceso del PP, estos juegan un rol central a la hora de dar viabilidad a las propuestas; en este punto se marca la distinción entre funcionarios más o menos afines a la gestión participativa.

Por otro lado, se ha generado una práctica muy positiva a los efectos de aprovechar los recursos para el PP, en concreto lo que se realiza es un estudio de las propuestas presentadas en función de los planes previstos por las diferentes áreas, y aquellos proyectos cuyo problema ya esté previsto solucionarse dentro de la actividad de algunas de las áreas de la Intendencia son valorados como inviables dentro del PP. En tercer lugar, al ser el PP un programa de la Intendencia, pero que para su ejecución necesita de la colaboración de otros órganos descentralizados, como los municipios y los Centros Comunales Zonales (CCZ), se genera un diálogo permanente y el desarrollo de colaboraciones tanto con el tercer nivel de gobierno como con los CCZ.

Finalmente, se destaca una coordinación más puntual con otro programa dependiente de la Asesoría de Desarrollo Municipal y Participación: WEBcinos, en donde a la enseñanza de informática se le agrega un módulo sobre cómo elaborar propuestas, de manera de generar una herramienta que les facilite la presentación en programas participativos como el PP.

Con relación a la capacidad del PP para orientar políticas públicas, se destacan las opiniones de que la política es más bien útil para resolver aspectos puntuales y no para desarrollar líneas de acción más generales. En lo que respecta a la utilización de las propuestas no financiadas, salvo algún caso puntual, los entrevistados coinciden en que no se las utiliza de ninguna forma: 
Yo mismo me he planteado que lo de desechar las propuestas que no salen votadas es una debilidad del PP (...) si esas obras no salieron en el PP, hay que trabajar para que esas obras igual se realicen si son importantes (participante del PP de Montevideo).

Según se manifiesta desde los funcionarios de la política, las excepciones estarían en algunas áreas como tránsito, en donde se toma nota de algunas problemáticas planteadas en el programa para incluirlas en su planificación.

Con relación a la vinculación con planes estratégicos, que es el otro elemento que la literatura menciona como favorable para impulsar a que los problemas abordados se vuelvan más sustantivos (Welp, 2015; Montecinos, 2009), Montevideo cuenta con un Plan Estratégico de Desarrollo Zonal (PLAEDES) elaborado participativamente, y de acuerdo con las entrevistas a los ciudadanos, se tiende a representar al PLAEDES como algo más profundo donde se discuten líneas más abstractas, en tanto que el PP desarrolla proyectos puntuales. Creen que hay una conexión, pero no aparece la idea de que el PLAEDES sea un orientador constante de los proyectos propuestos en el PP. Más bien aparece como un telón de fondo que al tener conceptos tan generales casi que todas las propuestas puntuales podrían vincularse de alguna forma, pero no se ve al PP como una herramienta para darle ejecución a algunas de las ideas de desarrollo planteadas en el PLAEDES. Tampoco se advierte una vinculación con otros planes estratégicos de la ciudad.

\subsubsection{Gualeguaychú}

El caso de Gualeguaychú acompaña la tendencia de las otras experiencias analizadas hasta aquí, donde se da una gran dispersión de propuestas pequeñas que involucra a muchos actores territoriales. A diferencia de las experiencias uruguayas, en Gualeguaychú se reserva la votación como forma de elegir propuestas para una última instancia, en caso de que no haya consenso en la asamblea final. Este mecanismo no ha sido utilizado nunca todavía y las definiciones se han tomado por acuerdo. Sin embargo, esta modalidad deliberativa no ha logrado, hasta ahora, disminuir la cantidad de propuestas y generar acuerdos para atacar problemáticas más grandes de la zona. Si bien el diseño habilita a que el total del dinero sea destinado a un solo proyecto, este punto no es ni siquiera planteado como una posibilidad. Por el contrario, los actores ya llegan a las asambleas con determinadas ideas para su organización y en el camino las van adaptando, pero siempre se continúa con la misma temática; si logran pasar los controles de viabilidad técnica, esta propuesta ingresará a la asamblea final, donde, en general, no se deja sin financiamiento ninguno de los proyectos presentes, pero para lograr no superar los montos impuestos por el gobierno para esa zona es necesario adaptar en la propia asamblea las propuestas, terminando en la mayoría de los casos con propuestas mucho más chicas que en la planificación original: "nosotros planteábamos originalmente la compra de ciento veinte sillas para el club, terminamos con cincuenta 
porque si no, no alcanzaba la plata, y todos tuvimos que bajar un poco para que nadie se fuera sin nada. Esto siempre es así (participante del PP de Gualeguaychú).

Con relación a la amplitud de las temáticas que se pueden plantear, se puede decir que es bastante abierto solamente dejando afuera obras de infraestructura de gran porte como pavimento, cordón cuneta, cloacas y agua. El argumento expresado por el gobierno para que estos temas no entren es que se dispone de programas de participación ciudadana específicos para trabajar esos temas y que sería imposible con esta metodología hacer frente a gastos de ese porte.

En el punto de la coordinación del PP con otras áreas se advierte, tanto por parte del responsable político como por una técnica del programa, que ese es un punto en el que se debe mejorar. Se percibe la burocracia estatal como un aspecto que choca con estos programas participativos que son más dinámicos y trasparentes. Se menciona concretamente la necesidad de coordinar con el área de planeamiento, buscando compartir información para potenciar el PP.

una buena experiencia fue la que una vez llevaron de Planeamiento a una zona, un proyecto integrado; bueno, lo mostraron con video, con todo, está divino, pero la gente ya había votado, ya tenían en mente otros proyectos; eso está bueno que se haga al inicio, mostrar lo que se está pensando, lo que se quiere hacer, para después ahí sí empezar a desarrollar un proyecto (técnica del PP de Gualeguaychú).

Desde el PP se tomó la decisión de que la ejecución del PP no pase por obra pública con el argumento de no quitarles recursos a otras áreas, pero al mismo tiempo esto les evitó someterse a algunas trabas. En general, el involucramiento de otras áreas fue valorado como muy bajo por quienes trabajan en PP.

Finalmente, en la capacidad del PP de orientar políticas públicas, si se toma como indicador el destino de los proyectos no aprobados, se puede considerar que, al igual que las otras experiencias analizadas, no tienen un destino institucionalizado de utilización, sí se menciona que algunos proyectos de más porte, que no se han podido viabilizar por el PP, se han tomado desde otras áreas, pero en general la capacidad de nutrir estas agendas no es muy grande. Se debe tener en cuenta que debido a la dinámica que ha adquirido la toma de decisiones, en general las propuestas que no salen aprobadas son solo aquellas que no tienen viabilidad. Se puede decir que el PP no está jugando un rol estratégico en el Municipio, sino que está cumpliendo una función de movilización con capacidad de resolver problemas muy puntuales, mientras que el resto de la gestión pasa por otro lado.

\subsubsection{Córdoba}

El PP de Córdoba inició la gestión 2011-2015 con un gran desafío que era recuperar la credibilidad de la herramienta tras un periodo de gestión al frente de Giacomino (Frente 
Cívico y Social), en donde el PP no había ejecutado las propuestas aprobadas, acumulando deudas con la ciudadanía. Este aspecto llevó a que la nueva gestión buscara hacerse cargo de la deuda anterior y luego pusiera en marcha un mecanismo de PP que resultara viable, de manera de no comprometerse en proyectos complejos que volvieran a generar incumplimientos. Esto generó una herramienta bastante acotada en cuanto a la amplitud temática, en donde se buscó consolidar algunas áreas en las que el Municipio estuviera en mayores condiciones de hacer frente, dejando fuera todas las demás problemáticas. Este se entendió desde la gestión de PP como un primer paso que debe en el futuro avanzar hacia nuevas temáticas, potenciando la herramienta y evitando la sobreinversión en los rubros iniciales.

Nosotros, al encontrarlo raquítico al PP, optamos por decir vamos por poco, y en ese poco pusimos cinco rubros que más o menos garantizaban la ejecución, porque el PP te puede poner un taller de bellas artes, de música clásica, pero si el municipio central no está preparado, capacitado para generar eso, corremos riesgo de causar una nueva frustración (responsable político del PP de Córdoba).

Tanto por parte de los ciudadanos como desde el Municipio, se advierte como un problema las limitaciones que colocan determinadas formalidades de la administración que llevan a que algunas organizaciones o barrios enteros asentados en condiciones irregulares no puedan tener ningún tipo de obra porque esto termina reforzando las desigualdades.

En lo que respecta a la coordinación entre el PP y otras áreas del gobierno, esta se visualiza como fundamental, ya que se trata de un programa que tiene presupuesto, pero que no tiene a cargo la ejecución. Se reconoce como una tarea difícil que está atravesada por la percepción y la valoración que cada área tiene sobre el PP, y se percibe que no basta con la voluntad del Intendente para que haya colaboración. O sea, se lo ve como un aspecto en el que se ha trabajado, pero que aún tiene mucho por mejorar.

Cada área tiene su propia percepción de lo que es el PP, a algunos no les interesa, otros no lo conocen, otros colaboran, otros no tanto (...) (técnico del PP).

Tuvimos que ir limando circuitos administrativos de ejecución del PP, yo creo que algo hemos avanzado (responsable político del PP).

En el tema de la orientación de políticas públicas no se visualiza una utilización sistemática de los proyectos no aprobados. Desde las autoridades del PP se mencionan algunos casos concretos en los que han logrado articulaciones generando emprendimientos en conjunto, por ejemplo, con el área de Cultura. La visión del gobierno es que tanto las Juntas de Participación Vecinal como el PP tienen un potencial ilimitado en este punto y que deben seguir profundizándolo. Sin embargo, entre los ciudadanos entrevistados se advierte con fuerza una visión positiva del PP como herramienta para resolver problemáticas concretas, pero entendiendo que la verdadera 
herramienta de cogestión es la Junta de Participación Vecinal y es a través de esta que deben darse las orientaciones a las áreas. Está muy difundida la idea de que el PP es una herramienta más con el que cuentan las Juntas de Participación Vecinal. Las que, por otro lado, han logrado un rol protagónico en el PP, siendo este muy poco plural en términos de participación.

\section{Consideraciones finales}

Las reflexiones sobre la base del análisis de la posición en el organigrama del gobierno local muestran que en general no se establecen contactos directos en el diagrama entre las áreas de planeamiento y presupuesto y los PP, por el contrario, en general estos se acercan al área de participación, mostrando una predisposición a pensar la herramienta más desde su contribución a la movilización ciudadana, que como una forma de gestionar participativamente los recursos públicos. La excepción, en este punto, sería Montevideo, donde el PP depende de una asesoría cuya ubicación le da mayor capacidad de ejercer una influencia transversal.

Con relación al alcance, pese a la variedad en los diseños -con más o menos deliberación, con lógicas ligadas a la competencia o al consenso en la toma de decisiones-, ninguna experiencia logra generar acuerdos lo suficientemente amplios como para trascender el espacio micro, de la cuadra o la organización, generando una multiplicidad de proyectos de impacto territorial y poblacional pequeños. La idea que parece subyacer es que más allá del diseño, ciudadanos y gobernantes se sienten cómodos en este nivel. El primero logra pequeños avances, pero muy próximos a su vida cotidiana, y el segundo genera participación, pero reservando determinados temas de más complejidad para resolver de manera tradicional. O sea, más allá de que algunos reglamentos dejan espacios para determinados acuerdos que podrían tender hacia la búsqueda de soluciones a problemas más generales de la zona, la apropiación subjetiva del diseño lleva a que los ciudadanos y el gobierno lo pongan en práctica de la manera que les resulta más funcional a su interés.

En lo que tiene que ver con la capacidad de orientar políticas públicas, se advierten limitaciones en las cuatro experiencias en este sentido, no logrando una forma institucionalizada de aprovechar aquellos proyectos que no obtienen financiación en el PP. Esta escasa capacidad de nutrir otras áreas va en consonancia tanto con la cercanía a los espacios de movilización como con la orientación micro de las propuestas.

Por último, la coordinación con otras áreas es fundamental en los PP, ya que estos habilitan espacios de inversión en muchos rubros que deben tener una cierta coherencia con lo planificado por el área. Estas colaboraciones también son en general percibidas como difíciles y atravesadas por la percepción predominante sobre el PP en las distintas áreas, siendo Montevideo la experiencia que cuenta con una mejor valoración en este 
punto. Si se vincula este aspecto con la propuesta de análisis de Melo Romão (2014), se puede sugerir que el estatus político del PP se vincula en estos casos con la localización en la administración municipal, siendo Montevideo la experiencia que tiene un PP con una localización más estratégica -ubicado cercano al Intendente y por fuera de la áreas específicas- el que, pese a no lograr trascender lo concreto y generar orientaciones hacia líneas de acción más generales, sí logra una mayor colaboración y un intercambio más fluido de información con los demás sectores de la Intendencia e incluso con el nivel municipal. Cabría plantearse, como una pregunta de indagación futura, si es la cercanía a las áreas de planeamiento y presupuesto o la cercanía al propio Intendente lo que le da una mayor articulación y capacidad de respuesta general al PP.

\section{Bibliografía}

Annunziata, R. (2011). La política de la singularidad de la experiencia. En Cheresky, I (Comp.). Ciudadanía y legitimidad democrática en América Latina. Buenos Aires: Prometeo. Annunziata, R. (2013). Democratizar la ciudad. Los Presupuestos Participativos en Rosario y Morón. Revista Nueva Sociedad, 243.

Cabannes, Y. (2004). Presupuesto Participativo y finanzas locales. Programa de Gestión Urbana. Coordinación para América Latina y El Caribe. BID. Ecuador.

Cabannes, Y. (2005). Presupuesto participativo y finanzas locales. Documento Base. Segunda versión ampliada. Red Urbal N 9: Alcaldía Municipal de Porto Alegre, Porto Alegre Recuperado en www.portoalegre.rs.gov.br/urbal (10/11/2015).

Goldfrank, B. (2011). Deepening local democracy in Latin America: participation, Decentralization and the left. University Park, Pennsylvania: The Pennsylvania State University Press.

Gurza Lavalle, A.; Melo Romão, W. y Zaremberg, G. (2014). Partidos políticos e innovación democrática: Más allá de las purezas y perversiones. Revista Mexicana de Ciencias Políticas y Sociales, Año LIX, 220. México: UNAM, pp. 21-54.

Martínez, C. y Arena, E. (2011). Sistematización de experiencias y buenas prácticas de Presupuesto Participativo. Documento preliminar. Buenos Aires: UNGS.

Melo Romão, W. P. B. (2014). For what? The political meaning of participatory budgeting. Chicago: LASA.

Molina, J. (2011). Los Presupuestos Participativos. Un modelo para priorizar objetivos y gestionar eficientemente en la administración local. España: Aranzadi.

Montecinos, E. (2009). El Presupuesto Participativo en América Latina. ¿Complemento o subordinación a la democracia representativa? Revista CLAD No 44-Reforma y Democracia. Caracas.

Montecinos, E. (2011). Democracia participativa y presupuesto participativo en Chile: ¿Complemento o subordinación a las instituciones representativas locales? Revista de Ciencia Política, 31, 1, pp. 63-89.

Montecinos, E. (2012). Diseños institucionales y participación ciudadana en presupuestos 
participativos: los casos de Chile, Argentina, Perú, República Dominicana y Uruguay. Comunicación presentada en XVII Congreso Internacional del CLAD sobre la Reforma del Estado y de la Administración Pública, Cartagena, Colombia, 30 oct.2 nov. 2012.

Rodgers, D. (2005). ¿Vale nada lo politizado? El Presupuesto Participativo de la ciudad de Buenos Aires, 2002-03. Crisis States Research Centre working papers series 1, 72. Crisis States Research. Centre, London School of Economics and Political Science, London, UK.

Schneider, C. y Welp, Y. (2011). ¿Transformación democrática o control político? Análisis comparado de la participación ciudadana institucional en América del Sur. Íconos, $\mathrm{N}^{\circ} 40$, pp. 21-39.

Sintomer, Y. (2008). Les budgets participatifs en Europe. Paris: La Découverte.

Welp, Y. (2015). Participación ciudadana, poder y democracia: apuntes para un debate. En RAPP (próxima publicación). Recuperado en https://www.academia.edu/10984935/Participaci\%C3\%B3n_ciudadana_poder_y_de mocracia_apuntes_para_un_debate (19/09/2016) 